\title{
Space-Time Trellis Code Construction for Fast Fading Channels
}

\author{
Zoltan Safar and K. J. Ray Liu \\ Department of Electrical and Computer Engineering \\ University of Maryland, College Park, MD 20742
}

\begin{abstract}
The need for bandwidth- and power-efficient wireless communication systems has raised considerable interest in spacetime codes. In this work, we propose a systematic space-time code construction procedure for fast fading channels. The method can be used to design space-time codes for an arbitrary number of transmit antennas and any memoryless modulation. We introduce a new design criterion that ensures full spatial diversity and develop the code design method based on this criterion. The flexibility of the proposed approach is demonstrated by designing spacetime trellis codes for 2,3 and 4 transmit antennas with QPSK, 8PSK and 4ASK modulations.
\end{abstract}

\section{INTRODUCTION}

In wireless communications, diversity techniques have been used extensively to improve the quality of transmission at high data rates. Spatial diversity corresponds to adding redundancy in the spatial domain: building a system with multiple transmit and/or receive antennas can improve the performance and throughput of the wireless link by making use of the large number of propagation paths between the transmitter and the receiver.

Space-time (ST) trellis codes represent a combination of forward error correction, transmit diversity and modulation. The performance criteria for both quasi-static and fast fading channels were derived in [1], characterizing the ST codes with two quantities: the diversity advantage, which describes the asymptotic error rate decrease as a function of the signal to noise ratio (SNR), and the coding advantage, which determines the vertical shift of the error performance curve.

Until recently, the focus of ST code research has been mainly on the quasi-static channel model. The authors of [1] proposed design rules for two transmit antennas to achieve the maximum diversity advantage. Later works [2], [3], [7] described systematic code design methods for an arbitrary number of transmit antennas.

The problem of code design for fast fading channels was first addressed in [4]. ST codes for 2 transmit antennas and QPSK modulation were designed using the idea of signal set partitioning. In [5], the design of ST codes for fast fading channels was also considered. The authors found ST codes for 2 transmit antennas and QPSK and 8PSK modulations through computer search.

In this paper, we develop a code construction procedure that jointly considers diversity advantage and coding advantage for an arbitrary number of transmit antennas and any memoryless constellation. Section II will introduce the notation and the mathematical model of the communication system. The relevant results of previous works will be briefly restated in Section
III. Section IV will describe a new design criterion and analyze the properties of the ST codes that satisfy this criterion. The code design method will be developed in Section V. Section VI will provide the simulation results, and some conclusions will be drawn in the last section.

\section{System Model}

Consider a wireless communications system with $K$ transmit and $L$ receive antennas. The input bit stream is divided into $b_{s}$ bit long blocks, forming $B$-ary $\left(B=2^{b_{s}}\right)$ source symbols. The ST encoder works as a finite state machine with $N$ states: it takes the current source symbol, $b_{t}\left(b_{t} \in\{0,1, \ldots, B-1\}\right)$, at discrete time $t$ and, governed by this input and the current state, $S_{t}\left(S_{t} \in\{0,1, \ldots, N-1\}\right)$, moves to the next state, $S_{t+1}$. During this state transition, the encoder outputs $K B$-ary channel symbol indices. We denote the channel symbol index for antenna $k, k=0,1, \ldots, K-1$, generated during the state transition from $S_{t}$ through the branch corresponding to source symbol $b_{t}$ by $i^{k}\left(S_{t}, b_{t}\right)$. We will also use the channel symbol index vector, defined as:

$$
\mathbf{i}\left(S_{t}, b_{t}\right)=\left[i^{0}\left(S_{t}, b_{t}\right), i^{1}\left(S_{t}, b_{t}\right), \ldots, i^{K-1}\left(S_{t}, b_{t}\right)\right]^{T} .
$$

These channel symbol indices are mapped onto channel symbols (or constellation points) by the modulators and transmitted through the transmit antennas. In the sequel, $c(i)$ will represent the constellation point corresponding to channel symbol index $i$. (For example, in case of $B$-ary PSK, $c(i)=\exp (j 2 \pi i / B$ ), where $j=\sqrt{-1}$.) All the constellations are assumed to be normalized so that the average energy of the constellation is unity (if the channel symbols are equally likely). $c\left(i^{k}\left(S_{t}, b_{t}\right)\right)$ will denote the constellation point output by antenna $k$ when the current state is $S_{t}$ and the current input is $b_{t}$. In vector notation:

$\mathbf{c}\left(S_{t}, b_{t}\right)=\left[c\left(i^{0}\left(S_{t}, b_{t}\right)\right), c\left(i^{1}\left(S_{t}, b_{t}\right)\right), \ldots, c\left(i^{K-1}\left(S_{t}, b_{t}\right)\right)\right]^{T}$.

The transmission medium is assumed to be a flat (frequency nonselective), fast Rayleigh fading channel. At each discrete time instant, the path gains between the transmit and the receive antennas are modeled as independent, complex, zero mean, circularly symmetric Gaussian random variables with unit variance. These path gains are assumed to be known by the receiver.

At the receiver side, the received signals at each receive antenna are demodulated, and the ST decoder produces the decoded bit stream. The receiver noise is modeled as independent, complex, zero mean, circularly symmetric Gaussian random variables. 


\section{PERFORMANCE CRITERIA}

Assume that the previously described transmitter sends $T$ $(T>K) B$-ary source symbols to the receiver. The ST encoder, while encoding the source symbol sequence $\left\{b_{t}\right\}$, goes through the sequence of states $\left\{S_{t}\right\}$, and produces $\mathbf{C}$, the $K$ by $T$ channel symbol matrix:

$$
\mathbf{C}=\left[\mathbf{c}\left(S_{0}, b_{0}\right), \mathbf{c}\left(S_{1}, b_{1}\right), \ldots, \mathbf{c}\left(S_{T-1}, b_{T-1}\right)\right] .
$$

The decoder, due to decoding errors, goes through a different sequence of states, $\left\{S_{t}^{\prime}\right\}$, producing the erroneously decoded source symbol sequence $\left\{b_{t}^{\prime}\right\}$ and the $K$ by $T$ channel symbol matrix $\mathbf{C}^{\prime}$ :

$$
\mathbf{C}^{\prime}=\left[\mathbf{c}\left(S_{0}^{\prime}, b_{0}^{\prime}\right), \mathbf{c}\left(S_{1}^{\prime}, b_{1}^{\prime}\right), \ldots, \mathbf{c}\left(S_{T-1}^{\prime}, b_{T-1}^{\prime}\right)\right] .
$$

Define $\mathbf{D}$, the channel symbol difference matrix, as $\mathbf{D}=\mathbf{C}-\mathbf{C}^{\prime}$. Assume that, for $\tau$ time instants $t_{0}, t_{1}, \ldots, t_{\tau-1}$ $\left(0 \leq t_{i} \leq T-1\right)$, the $t_{i}$ th column of $\mathbf{D}$ is nonzero, i.e.

$$
\mathbf{c}\left(S_{t_{i}}, b_{t_{i}}\right)-\mathbf{c}\left(S_{t_{i}}^{\prime}, b_{t_{i}}^{\prime}\right) \neq \mathbf{0}, \quad \text { for } i=0,1, \ldots, \tau-1 .
$$

Given the earlier described channel model, the probability that the decoder erroneously decodes $\mathbf{C}^{\prime}$ if $\mathbf{C}$ was sent can be upper bounded as [1]:

$P\left(\mathbf{C}^{\prime} \mid \mathbf{C}\right) \leq\left(\frac{E_{0}}{4 K N_{0}}\right)^{-\tau L} \cdot \prod_{i=0}^{\tau-1} \frac{1}{\left\|\mathbf{c}\left(S_{t_{i}}, b_{t_{i}}\right)-\mathbf{c}\left(S_{t_{i}}^{\prime}, b_{t_{i}}^{\prime}\right)\right\|^{2 L}}$

where $E_{0} / N_{0}$ is the average signal to noise ratio per source symbol at the receive antennas and $\|\mathbf{x}\|=\sqrt{\mathbf{x}^{H} \mathbf{x}}$. The performance criteria [1] were derived to minimize $P\left(\mathbf{C}^{\prime} \mid \mathbf{C}\right)$ :

1. Design for diversity advantage (distance criterion): The minimum number of nonzero columns in $\mathbf{D}$ (the number $\tau$ ) taken over all distinct $\mathbf{C}$ and $\mathbf{C}^{\prime}$ matrices must be maximized.

2. Design for coding advantage (product criterion): The minimum of the norm products

$$
\delta=\prod_{i=0}^{\tau-1}\left\|\mathbf{c}\left(S_{t_{i}}, b_{t_{i}}\right)-\mathbf{c}\left(S_{t_{i}}^{\prime}, b_{t_{i}}^{\prime}\right)\right\|^{2}
$$

taken over all distinct $\mathbf{C}$ and $\mathbf{C}^{\prime}$ matrices must be as large as possible.

\section{A New Design CRiterion}

For ST encoders having $N=B^{K-1}$ states, we propose a new design criterion that is based on the distance criterion described above. The design criterion (uniqueness criterion) is: Every channel symbol index vector must be unique. That is:

(a) The channel symbol index vectors assigned to different branches emanating from the same state must be different.

(b) Any channel symbol index vector assigned to any state must be different from any channel symbol index vector assigned to any other state.
Each channel symbol index vector contains $K B$-ary symbols, so there are $B^{K}$ different channel symbol index vectors. Since there are $B$ branches emanating from each state and the encoder is assumed to have $B^{K-1}$ states, we need exactly $B^{K}$ different channel symbol index vectors. Therefore, it is possible to assign channel symbol index vectors to state transitions according to the uniqueness criterion.

The following two subsections will analyze the properties of the ST codes that satisfy the proposed criterion.

\section{A. Diversity Advantage}

Without loss of generality, we can assume that the first decoding error occurs at $S_{0}$, so the correct and decoded paths diverge at this point (i.e. $S_{0}=S_{0}^{\prime}$ and $b_{0} \neq b_{0}^{\prime}$ ). The two paths are assumed to merge later at time $t=\tau$. As a consequence of criterion (a), the channel symbol vectors corresponding to the two paths diverging at $S_{0}$ will be different. Moreover, as a result of criterion (b), the the channel symbol vectors corresponding to the correct and the decoded paths going through different states will be different. Therefore, $\mathbf{D}_{\tau}$, the channel symbol difference matrix corresponding to any $\tau$-long error event (the first $\tau$ columns of $\mathbf{D})$, given by

$\mathbf{D}_{\tau}=\left[\mathbf{c}\left(S_{0}, b_{0}\right)-\mathbf{c}\left(S_{0}^{\prime}, b_{0}^{\prime}\right), \ldots, \mathbf{c}\left(S_{\tau-1}, b_{\tau-1}\right)-\mathbf{c}\left(S_{\tau-1}^{\prime}, b_{\tau-1}^{\prime}\right)\right]$

will have $\tau$ nonzero columns. From the performance criteria described in Section III, one can conclude that any $\tau$-long error event will achieve a diversity advantage of $\tau L$. In case of multiple error events, the total diversity advantage will be the sum of the diversity advantages of the individual error events. Consequently, the the minimum diversity advantage of the ST code is determined by the shortest error event. In case of ST encoders having $B^{K-1}$ states, the shortest error event is $K$ state transitions long [7], so the ST code is guaranteed to achieve a diversity advantage of $K L$.

\section{B. Node Error Probability}

In this section, we will derive an upper bound on the node error probability (the probability that at any given discrete time instant, a decoding error occurs, making the correct and the decoded paths diverge). For the purpose of the analysis, we will assume that the trellis is infinite.

Using the union bound, the node error probability can be upper bounded as:

$$
P_{e} \leq \sum_{\mathbf{C}} \sum_{\tau=K}^{\infty} \sum_{\mathbf{C}^{\prime}{ }_{\tau}} P\left(\mathbf{C}_{\tau}^{\prime} \mid \mathbf{C}\right) P(\mathbf{C})
$$

In (2), the first summation is over all possible sent channel symbol matrices $\mathbf{C}$, the second summation is over all possible error event lengths, and the third is over all possible erroneously decoded channel symbol matrices $\mathbf{C}_{\tau}^{\prime}$ corresponding to $\tau$-long error events. Assuming that the decoding error occurs at state $S_{0}$, the columns of $\mathbf{D}_{\tau}$ will be nonzero, i.e. for $t=0,1, \ldots, \tau-1$, at least one coordinate of the channel symbol vectors $\mathbf{c}\left(S_{t}, b_{t}\right)$ 
and $\mathbf{c}\left(S_{t}^{\prime}, b_{t}^{\prime}\right)$ will be different. Therefore, if we denote the minimum distance of the chosen constellation by $\Delta$, the squared norms of the columns of the $\mathbf{D}_{\tau}$ matrix can be lower bounded as

$$
\left\|\mathbf{c}\left(S_{t}, b_{t}\right)-\mathbf{c}\left(S_{t}^{\prime}, b_{t}^{\prime}\right)\right\|^{2} \geq \Delta^{2} \quad \text { for } t=0,1, \ldots, \tau-1 .
$$

Substituting (3) into (1), we obtain an upper bound on the pairwise error probability:

$$
P\left(\mathbf{C}_{\tau}^{\prime} \mid \mathbf{C}\right) \leq\left(\frac{E_{0}}{4 K N_{0}}\right)^{-\tau L} \Delta^{-2 \tau L}
$$

Since the upper bound (4) does not depend on $\mathbf{C}_{\tau}^{\prime}$, (2) can be rewritten as:

$$
P_{e} \leq \sum_{\mathbf{C}} P(\mathbf{C}) \sum_{\tau=K}^{\infty} N\left(\mathbf{C}_{\tau}^{\prime}\right)\left(\frac{E_{0} \Delta^{2}}{4 K N_{0}}\right)^{-\tau L}
$$

where $N\left(\mathbf{C}_{\tau}^{\prime}\right)$ is the number of $\tau$-long error paths for a given correct path. $N\left(\mathbf{C}_{\tau}^{\prime}\right)$ can be strictly upper bounded by $B^{\tau}$, so the upper bound simplifies to:

$$
P_{e}<\sum_{\mathbf{C}} P(\mathbf{C}) \sum_{\tau=K}^{\infty} B^{\tau}\left(\frac{E_{0} \Delta^{2}}{4 K N_{0}}\right)^{-\tau L} .
$$

Finally, recognizing that the terms on the right side of the summation over $\tau$ do not depend on $\mathbf{C}$ and that the probability mass function of $\mathbf{C}$ sums to unity, the final expression for the upper bound on the node error probability becomes:

$$
P_{e}<\sum_{\tau=K}^{\infty}\left(\frac{E_{0} \Delta^{2}}{4 K N_{0} \sqrt[L]{B}}\right)^{-\tau L}
$$

From (7), one can see that the probability that a $\tau$ state transitions long error event occurs (the probability of the union of the $\tau$-long error events) decreases at least exponentially with $\tau$. Consequently, at high SNR, the shortest error events will dominate; the longer error paths will have negligible contribution to the node error probability.

\section{THE DESIGN METHOD}

In the $\mathrm{ST}$ encoder, $B$ channel symbol index vectors are assigned to each state, according to the branches emanating from that state. The current source symbol selects one of them, and the $k$ th $(k=0,1, \ldots, K-1)$ index of the chosen vector determines the constellation point for antenna $k$. Figure 1 depicts an example ST code for 3 antennas and any 4-ary constellation ( $K=3, b=2, N=16$ ). In this case, if the current state is state 2 and the value of the current source symbol is 3 , the ST encoder selects the 3 rd channel symbol index vector, $[1,3,1]^{T}$, and moves to state 11 . The 0 th, 1 st and 2 nd antennas will transmit the channel symbols corresponding to the indices 1,3 and 1, respectively.

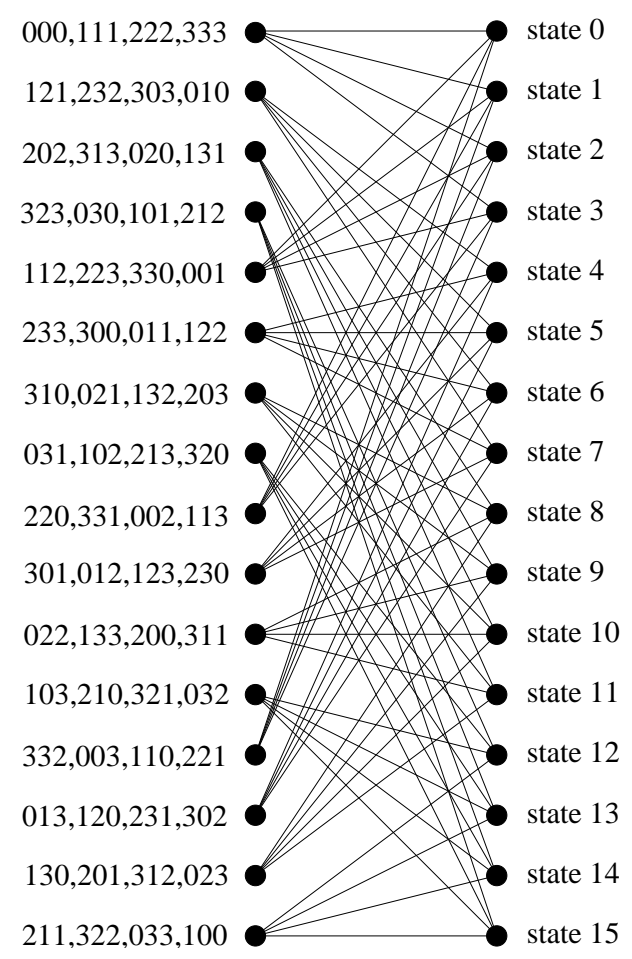

Fig. 1. Example ST code for 3 antennas, 4-ary modulation

This section addresses the problem of assigning channel symbol index vectors to state transitions. The most important objective is to maximize the diversity advantage, so the ST codes are required to satisfy the uniqueness criterion. The remaining freedom can be used to increase the value of the minimum norm product $(\delta)$. Since the available $B^{K}$ channel symbol index vectors can be arranged in $\left(B^{K}\right)$ ! different ways, the computational complexity of exhaustive search becomes prohibitive, as the number of transmit antennas and the constellation size increase. Therefore, we propose an approach that does not guarantee optimality, but is simple and flexible. The basic idea behind the method is that it attempts to maximize the minimum norm product corresponding to the shortest error events by maximizing the number of nonzero entries in the channel symbol difference matrix. Since the shortest error events achieve the minimum diversity advantage, it is a reasonable objective to maximize the norm product of these error events. Moreover, it was shown in Section IV-B that at higher SNR, the shortest error events will dominate the performance of the ST codes.

Since the $\mathrm{ST}$ encoder has $B^{K-1}$ states, any state $S(S \in$ $\left.\left\{0,1, \ldots, B^{K-1}-1\right\}\right)$ can be uniquely represented as a $K-1$ digit $B$-ary number with digits $l_{1}, l_{2}, \ldots, l_{K-1}\left(l_{k} \in\right.$ $\{0,1, \ldots, B-1\})$ :

$$
S=B^{K-2} l_{K-1}+B^{K-3} l_{K-2}+\ldots+B l_{2}+l_{1} .
$$

The proposed design rules are:

1. The 0th index of the channel symbol index vectors (the channel symbol indices for the 0th transmit antenna) corre- 
sponding to input $b(b \in\{0,1, \ldots, B-1\})$ at state $S$ is determined as:

$$
i^{0}(S, b)=\left(b+l_{K-1}+l_{K-2}+\ldots+l_{1}\right) \bmod B .
$$

2. The rest of the indices (the channel symbol indices for the rest of the transmit antennas) are calculated as:

$i^{k}(S, b)=\left(i^{0}(S, b)+l_{k}\right) \bmod B \quad$ for $k=1,2, \ldots, K-1$.

It can be verified that the ST codes produced by the above described construction method satisfy the uniqueness criterion for any $B$-ary constellation.

As an example, consider the ST code shown in Figure 1. Since $B=4$, state $S=6$ can be represented as $S=B l_{2}+l_{1}$ with $l_{2}=1$ and $l_{1}=2$. The channel symbol indices corresponding to the 2 nd branch $(b=2)$ emanating from state 6 are determined as:

$$
\begin{gathered}
i^{0}(S, b)=\left(b+l_{2}+l_{1}\right) \bmod B=1, \\
i^{1}(S, b)=\left(i^{0}(S, b)+l_{1}\right) \bmod B=3, \\
i^{2}(S, b)=\left(i^{0}(S, b)+l_{2}\right) \bmod B=2 .
\end{gathered}
$$

Therefore, the channel symbol index vector assigned to this state transition will be $[1,3,2]^{T}$.

Assume that the correct and the erroneously decoded paths diverge at $S_{0}$ and merge at $S_{K}$. Thus, $S_{0}=S_{0}^{\prime}, b_{0} \neq b_{0}^{\prime}$, and $S_{K}=S_{K}^{\prime}$. The following theorem characterizes $\mathbf{D}_{K}$, the channel symbol difference matrix corresponding to the shortest error events (the first $K$ columns of $\mathbf{D}$ ), given by

$$
\begin{aligned}
& \mathbf{D}_{K}=\left[\mathbf{c}\left(S_{0}, b_{0}\right)-\mathbf{c}\left(S_{0}^{\prime}, b_{0}^{\prime}\right), \ldots\right. \\
& \left.\ldots, \mathbf{c}\left(S_{K-1}, b_{K-1}\right)-\mathbf{c}\left(S_{K-1}^{\prime}, b_{K-1}^{\prime}\right)\right],
\end{aligned}
$$

in case of the proposed design rules, for any $B$-ary modulation.

Theorem 1: For any $B \geq 2$, the 0 th column of the $\mathbf{D}_{K}$ matrix contains only nonzero entries. If $B=2$ (binary modulation), the diagonal elements of $\mathbf{D}_{K}$ in the $1 \mathrm{st}, 2 \mathrm{nd}, \ldots,(K-1) \mathrm{st}$ columns will be zero, the rest of the elements will be nonzero. If $B>2$ (higher order modulation) and $b_{0}^{\prime}=\left(b_{0}+B / 2\right) \bmod B$, the the diagonal elements of $\mathbf{D}_{K}$ in the $1 \mathrm{st}, 2 \mathrm{nd}, \ldots,(K-1) \mathrm{st}$ columns will be zero, the rest of the elements will be nonzero. For each correct path, there is exactly one such $K$-long error path. If $B>2$ and $b_{0}^{\prime} \neq\left(b_{0}+B / 2\right) \bmod B$, the matrix $\mathbf{D}_{K}$ will contain only nonzero entries. For each correct path, there are exactly $B-2$ such $K$-long error paths.

\section{Simulation Results}

To illustrate the performance of the codes designed using the above described method, we present some simulation results. The simulated communication system had one receive antenna. The source symbols were transmitted in frames of length 130, and the Viterbi algorithm with decoding depth of 20 state transitions was used to decode the received signals. For each discrete time instant, the path gains between the transmit antennas
TABLE I

MINIMUM NORM PRODUCTS FOR 2 ANTENNAS, QPSK

\begin{tabular}{|c||c|c|c|c|c|}
\hline$\tau$ & 2 & 3 & 4 & 5 & 6 \\
\hline$\delta_{\tau}$ & 24 & 32 & 64 & 128 & 256 \\
\hline$\delta_{\tau}[4]$ & 16 & 32 & 96 & 128 & 256 \\
\hline
\end{tabular}

TABLE II

MINIMUM NORM PRODUCTS FOR 3 ANTENNAS, QPSK

\begin{tabular}{|c||c|c|c|c|}
\hline$\tau$ & 3 & 4 & 5 & 6 \\
\hline$\delta_{\tau}$ & 384 & 384 & 2304 & 6144 \\
\hline$\delta_{\tau}[4]$ & 216 & 576 & 1728 & 2880 \\
\hline
\end{tabular}

and the receive antenna were modeled as independent, complex, zero mean, circularly symmetric Gaussian random variables with unit variance.

Since the frame error probability depends on the length of the frame, we present probability of bit error curves as functions of the average signal to noise ratio (SNR) per source symbol at the receive antenna.

Figure 2 depicts the performance of the ST codes designed for 2 transmit antennas and QPSK constellation $(K=2, B=$ $4, N=4)$. We compare our method with the ST codes of [4], [5] and [6]. All of these codes satisfy the uniqueness criterion, even the ST code given in [6], despite the fact that this code was designed for the quasi-static fading channel model. It is observed that our method produced a ST code that has the same performance as the ST code of [5], which was found by exhaustive search.

The theoretical performance of the above codes was also compared. Table I shows the minimum norm product values $\delta_{\tau}$ for the $\tau$-long error events. The entries in the second row of the table correspond to the ST codes of [4], [5] and our method, while the third row contains the $\delta_{\tau}$ values for the ST code of [6]. The table entries were obtained by performing computer search. These $\delta_{\tau}$ values predict the similar performance of the methods described in [4], [5] and the proposed approach, along with the slightly worse performance of the ST code provided in [6] at high SNR.

The bit error rate curves for 3 transmit antennas and QPSK modulation ( $K=3, B=4, N=16)$ are depicted in Figure 3. We have not been able to find ST codes designed for more than 2 transmit antennas and fast fading channels, so we compare our method (the code given in Figure 1) with the ST code described in [6]. This ST code also satisfies the uniqueness criterion. The curves show that our ST code outperforms the ST code of [6] at higher SNR.

The minimum norm product values for the proposed method are given in the second row of Table II, and the third row contains the values for the ST code of [6]. The theoretical norm product values confirm the tendencies observed in Figure 3.

Finally, Figures 4 and 5 show the performance of the ST codes designed by our approach for 3 transmit antennas and 


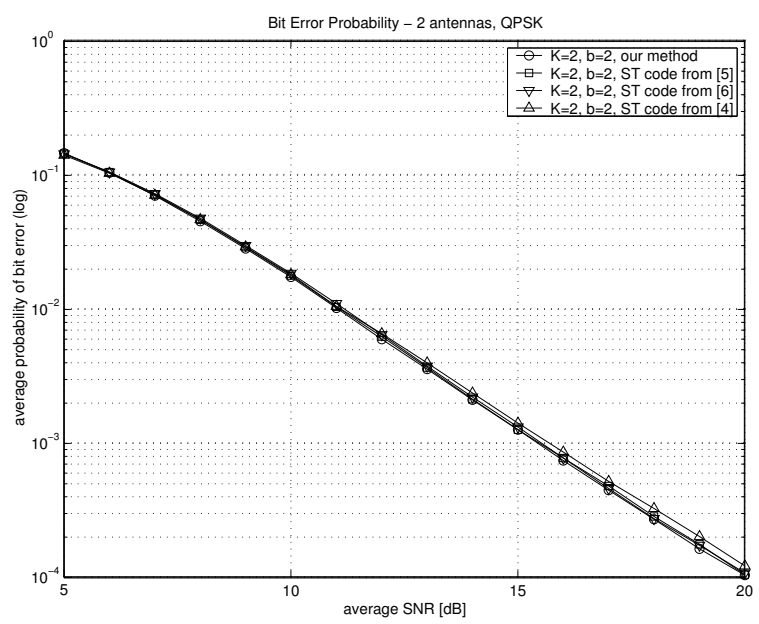

Fig. 2. ST codes for 2 antennas, QPSK

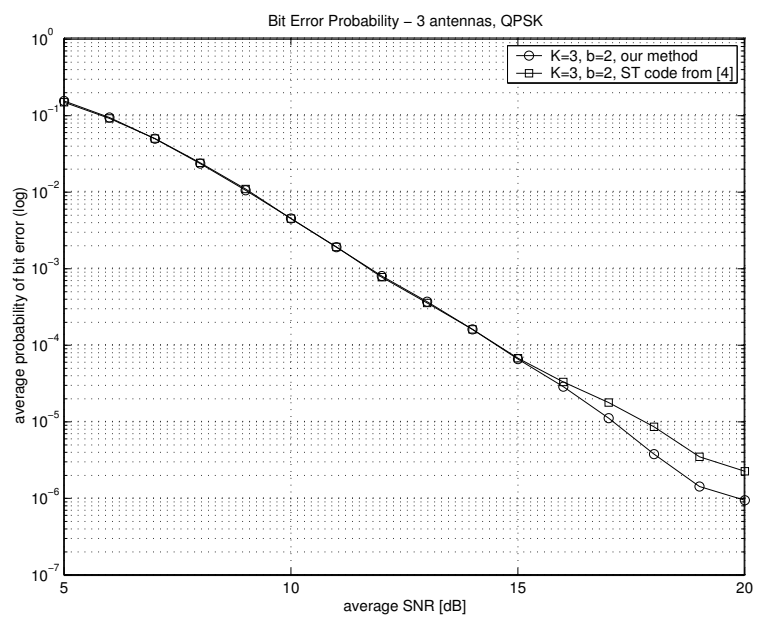

Fig. 3. ST codes for 3 antennas, QPSK

8PSK modulation ( $K=3, B=8, N=64$ ), and 4 antennas and 4ASK modulation $(K=4, B=4, N=64)$, respectively. To the best of our knowledge, no ST codes have been published that we could compare against.

As discussed in Section IV-A, the achieved diversity advantage depends on the length of the decoding error events. As the SNR increases, the average length of the decoding error events decreases. Therefore, the steepness of the error performance curve is expected to decrease at higher SNR. This phenomenon can be observed in Figures 3 and 5 .

\section{CONCLUSION}

We proposed a new ST code design criterion for fast Rayleigh fading channels and analyzed its properties. Based on this criterion, we developed a systematic ST trellis code design method for an arbitrary number of transmit antennas and any memoryless modulation. Even though the proposed method does not guarantee optimality, both the theoretical minimum norm product values and the simulations show that our system-

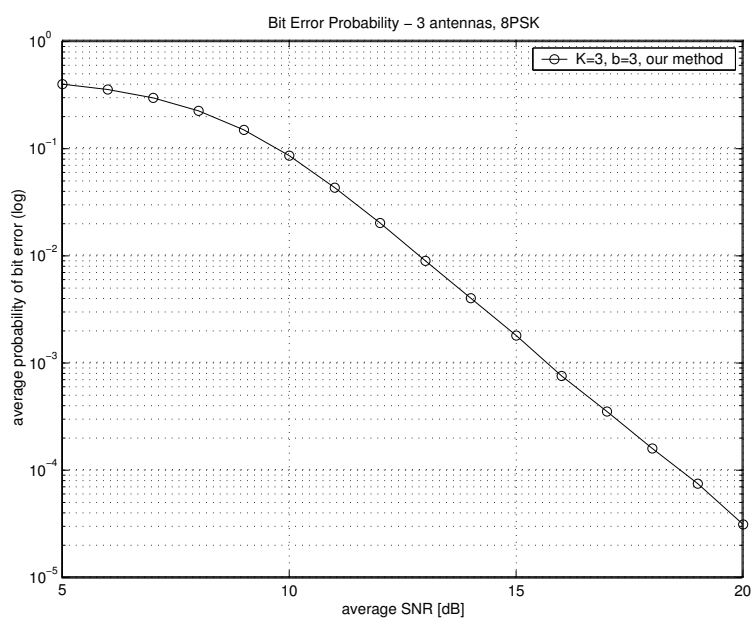

Fig. 4. ST code for 3 antennas, 8PSK

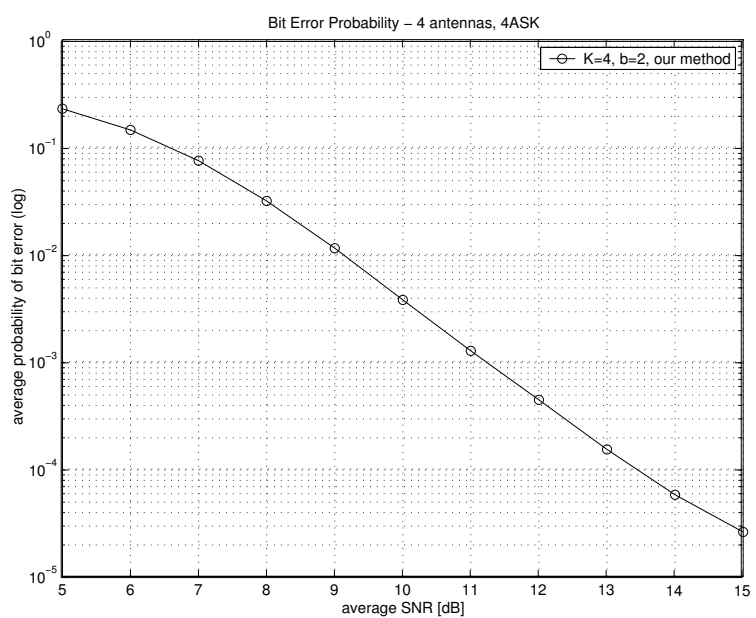

Fig. 5. ST code for 4 antennas, 4 ASK

atic design procedure results in ST codes that perform very well compared to the ones previously found by computer search.

\section{REFERENCES}

[1] V. Tarokh, N. Seshadri, A. Calderbank, Space-Time Codes for High Data Rate Wireless Communication: Performance Criterion and Code Construction, IEEE Transactions on Information Theory, Vol. 44, No. 2, pp. 744-765, March 1998.

[2] J. Grimm and M. Fitz and J. Krogmeier, Further Results on Space-Time Coding for Rayleigh Fading, Proceedings of the 36th Allerton Conference on Communications, Control and Computing, pp. 391-400, 1998.

[3] A. R. Hammons and H. El Gamal, On the Theory of Space-Time Codes for PSK Modulation, IEEE Transactions on Information Theory, Vol. 46, No. 2, pp. 524-542, March 2000.

[4] S. Zummo and S. Al-Semari, Space-Time Coded QPSK for Rapid Fading Channels, Proceedings of PIMRC, Vol. 1, pp. 504-508, 2000.

[5] W. Firmanto, B. Vucetic and J. Yuan, Space-Time TCM with Improved Performance on Fast Fading Channels, IEEE Communications Letters, Vol. 5, No. 4, pp. 154-156, April 2001.

[6] Q. Yan and R. Blum, Optimum Space-Time Convolutional Codes, Proceedings of WCNC, Vol. 3, pp. 1351-1355, 2000.

[7] Z. Safar and K.J.R. Liu, Systematic Design of Space-Time Trellis Codes for Diversity and Coding Advantages, to appear, EURASIP Journal on Applied Signal Processing, Special Issue on Space-Time Coding and Its Applications, March 2002. 